\title{
Estrategias de intervención en la UCI neonatal: un enfoque fisioterapéutico
}

Laura Valentina López Rodríguez *

\begin{abstract}
*Fisioterapeuta con énfasis clínico. Universidad del Rosario. Bogotá. Colombia.
Correspondencia: FT. Laura Valentina López Rodríguez. Escuela de Medicina y ciencias de la salud. Universidad del Rosario. Ak. 24 \#\#63C-69, Bogotá, Colombia. Teléfono: 57 2970200. Correo electrónico: lauraval.lopez@urosario.edu.co
\end{abstract}

Resumen

El fisioterapeuta a lo largo de su profesión ha ampliado el espectro de intervenciones y escenarios de desempeño, siendo uno de los más recientes el abordaje en la unidad de cuidado intensivo neonatal. Recientemente se han reportado en la literatura diferentes estrategias de intervención con el objetivo de lograr un desarrollo adecuado del neonato, tales como masaje, estimulación kinestésica, educación en el programa madre canguro, y maniobras de tórax. El objetivo del artículo fue revisar la información actual proveniente de la evidencia científica disponible sobre estas diferentes estrategias de intervención aplicadas en la unidad de cuidado intensivo neonatal, por lo que se realizó una revisión bibliográfica de los artículos encontrados entre febrero y agosto del 2019 en las bases de datos PUBMED y SCOPUS. Se obtuvieron 40 artículos que cumplieron los criterios de selección y que se incluyeron en la revisión. Finalmente, se concluye que estas estrategias de intervención fisioterapéutica contribuyen al logro de un desarrollo integral adecuado en el neonato. MÉD.UIS.2021;34(1): 63-72

Palabras clave: Cuidado Intensivo Neonatal. Masaje. Método madre canguro. Estimulación Física. Terapia Respiratoria. Maniobras de tórax. Desarrollo neuromotor.

\section{Intervention strategies in the neonatal ICU: a physiotherapeutic approach}

\begin{abstract}
The physiotherapist throughout his profession has broadened the spectrum of interventions and performance scenarios, one of the most recent being the approach in the neonatal intensive care unit. Recently, different intervention strategies have been reported in the literature with the aim of achieving adequate development of the newborn, such as massage, kinesthetic stimulation, education in the kangaroo mother program, and chest maneuvers. This article aimed to review the current information from the scientific evidence available on these different intervention strategies in the neonatal intensive care unit, so a bibliographic review of the articles found between February and August 2019 in PUBMED and SCOPUS databases was carried out. 40 articles which met the eligibility criteria were obtained and included in the review. Finally, it is concluded that these physiotherapeutic intervention strategies contribute to the achievement of an adequate integral development in the neonate. MÉD.UIS.2021;34(1): 63-72
\end{abstract}

Keywords: Neonatal Intensive Care. Massage. Kangaroo mother care. Physical stimulation. Respiratory Therapy. Chest physiotherapy. Neuromotor development. 
¿Cómo citar este artículo?: López Rodríguez LV. Estrategias de intervención en la UCI neonatal: un enfoque fisioterapéutico. MÉD.UIS.2021;34(1):63-72 doi: 10.18273/revmed.v34n1-2021007

\section{Introducción}

A lo largo de la historia y el desarrollo de la fisioterapia como profesión, se ha evidenciado la variedad de enfoques que la práctica de esta posee. Uno de los más recientes es el abordaje del neonato en la Unidad de Cuidados Intensivos Neonatal (UCIN), el cual ha venido presentando un crecimiento continuo y acelerado en Latinoamérica, particularmente en las dos últimas décadas favoreciendo el surgimiento de preguntas sobre lo que se hace en la práctica o su función final en el paciente 1 .

Este crecimiento se ha visto relacionado y favorecido por el implemento de estrategias por parte del gobierno colombiano desde el año 2012 para reducir la mortalidad neonatal, logrando con estas que para el 2017 más del 96,5\% de los partos fueran atendidos institucionalmente por personal calificado, con mejores cuidados, y con implementación de medidas preventivas y oportunas de manejo en hospitalización o en unidades de cuidado intensivo neonatal, dependiendo la necesidad del neonato ${ }^{2}$.

Por otra parte, en un estudio retrospectivo que incluyó recién nacidos ingresados a la UCIN entre el 1 de enero y el 31 de diciembre de 2016, en una empresa prestadora de salud colombiana localizada en 12 departamentos del país, se encontró que el $45.5 \%$ de los recién nacidos ingresados eran neonatos prematuros ${ }^{3}$. Adicionalmente, se encontró que la mayor frecuencia en hospitalización de la UCIN ocurrió por enfermedades del tracto respiratorio, principalmente por enfermedad de membrana hialina, bronquiolitis y taquipneas transitorias del recién nacido, asociadas a la mala adaptación pulmonar al momento del nacimiento ${ }^{2-4}$. Estas alteraciones conllevan a que se genere un alto ingreso de pacientes a la UCIN, lo cual se correlaciona con la necesidad de requerimientos de oxígeno suplementario o de ventilación mecánica, además de un posible aumento en los días de estancia hospitalaria3,5. Esto dilucida la importancia de las diferentes estrategias de intervención como la fisioterapéutica en neonatos durante su estancia en la UCIN, ya que son de gran relevancia al favorecer el cuidado y manejo integral de este ${ }^{2-5}$. Es importante aclarar que la información disponible encontrada durante la búsqueda, de parte de las diferentes instituciones, es escasa sobre este tema.

De igual manera, se observa que la práctica fisioterapéutica se guía en el modelo sinactivo del desarrollo infantil, en el que los sistemas musculoesquelético, nervioso, comportamental y sensorial presentan múltiples dimensiones que se interrelacionan y que se organizan como subsistemas y afectan el desarrollo del infante. Estos subsistemas, a su vez, incluyen los sistemas autónomo, motor, organizativo, de atención interactiva y el de autorregulación ${ }^{6-8}$. Con base en esta concepción, el fisioterapeuta observa y evalúa si los subsistemas funcionan de la forma adecuada y de no ser así, realiza estrategias e intervenciones para lograrlo ${ }^{8,9}$.

En cuanto a las estrategias, a nivel global se encuentra que las más utilizadas son estimulación kinestésica, aplicada a aproximadamente un $70 \%$ de neonatos, seguida del método madre canguro y de masaje con un $40 \%$, mientras que las técnicas respiratorias son muy variables debido a la especificidad de las condiciones en las que debe aplicarse ${ }^{5}$. Aún así, en general, según lo que se ha encontrado en la evidencia, la práctica del masaje, la estimulación kinestésica, la educación a padres sobre el método madre canguro, las maniobras de tórax y las técnicas de digitopresión son las estrategias más usadas.

El masaje se define como la aplicación de una técnica manual que incluye tacto, effleurage, fricción, vibración, percusión, estiramiento y compresión al tejido superficial de la piel, músculo, tendón, ligamento y fascia ${ }^{10}$. Esta técnica se puede aplicar en neonatos prematuros mayores de 32 semanas ${ }^{11-13}$; sin embargo, algunos estudios han documentado que también se puede realizar en recién nacidos desde las 30 semanas con un peso mayor a 1000 gramos $^{13-18}$. En ambos casos mencionados, los bebés deben presentar signos vitales estables, no deben de cursar con procesos infecciosos o alteraciones en la piel, ni con anomalías congénitas importantes como trastornos neuromusculares, cardiopatías congénitas, defectos del tubo neural, y malformaciones gastrointestinales, tampoco deben cursar con hemorragia intraventricular de grado tres $y$ superior; finalmente, no deben de tener restricciones 
en su movimiento por fracturas y/o deformidades óseas, ni se debe realizar en neonatos que hayan presentado cirugías recientes ${ }^{12,19}$. Si el neonato presenta los criterios para realizar la técnica, se deben monitorizar continuamente los signos vitales, y tener precaución y vigilancia ante posibles efectos adversos como apneas, desaturación de oxígeno, bradicardia y taquicardia ${ }^{18}$. La aplicación adecuada de esta técnica ha evidenciado beneficios en cuanto a ganancia de peso y de talla ${ }^{10,12,20,21}$, regulación del sueño ${ }^{17,22}$ y de parámetros cardiovasculares ${ }^{23}$, control del dolor ${ }^{24}$, desarrollo del sistema motor ${ }^{16,25,26}$, del sistema inmune ${ }^{18}$, y de la función gastrointestinal ${ }^{19,27}$.

Adicionalmente, se encuentra reportada en la literatura la estimulación kinestésica, la cual consiste en la aplicación de movimientos articulares pasivos y la facilitación de movimientos espontáneos dentro de los rangos normales de movimiento, que permiten el desarrollo de habilidades motoras en los niños prematuros ${ }^{6,28}$; todo esto debido a que las vías motoras que forman los tractos corticoespinales y la mielinización dependen de la actividad metabólica que se estimula con el movimiento ${ }^{29}$. Los criterios para poder realizar la estimulación kinestésica son similares al de otras maniobras como el masaje, con la excepción de que los estudios recomiendan realizar la técnica 30 a 60 minutos después de la alimentación, para así lograr una adecuada digestión y evitar reflujo gástrico ${ }^{28,30}$. Finalmente, en neonatos con sospecha de lesión cerebral, la estimulación debe ser realizada por un experto, con la cabeza en posición neutral y con una elevación de $30^{\circ}$ durante la intervención para prevenir aumento de la presión intracraneana ${ }^{31}$. La aplicación adecuada de la técnica ha reportado efectos benéficos en cuanto a mejoría en la mineralización ósea, eficiencia metabólica y en cuanto al posible logro de respuestas conductuales y motoras optimas que conlleven a un desarrollo sensorio motriz adecuado ${ }^{6,14,29}$. Recientemente, se han reportado efectos beneficiosos en el desarrollo neuromotor con la combinación de masaje y estimulación kinestésica; algunos autores han incluido esta última dentro de la definición de masaje y en la práctica clínica se han empezado a implementar simultáneamente ${ }^{16}$.

Por otra parte el Método Madre Canguro (MMC) es un sistema de cuidados del niño prematuro y/o de bajo peso al nacer, estandarizado y protocolizado basado en el contacto piel a piel entre el niño y sus padres ${ }^{32,33}$, tanto en el hospital como después del alta temprana, hasta al menos la semana 40 de edad gestacional postnatal con lactancia materna idealmente exclusiva y seguimiento adecuado ${ }^{34}$. Iniciado por el Dr. Edgar Rey en 1978 en el Instituto Materno Infantil en Bogotá, Colombia33, para esta técnica el bebé debe ser vestido con un pañal y una gorra, y ser sostenido en posición vertical contra el pecho desnudo de la madre y cubierto en el dorso con la ropa materna ${ }^{35}$.

Este método reduce la mortalidad neonatal en niños con bajo peso al nacer y prematuros a nivel mundial ${ }^{36}$, y desde una perspectiva psicológica, se cree que es crucial para desarrollar estrategias biológicas, mentales y socio conductuales de un neonato prematuro en su entorno. Los mecanismos fisiológicos por los cuales se logran los efectos beneficiosos aún se encuentran en estudio, sin embargo, la literatura reporta que los receptores cutáneos, musculoesqueléticos, viscerales y vestibular-cocleares, que se proyectan a los centros cerebrales del neonato y participan en el control respiratorio y en el flujo de salida vagal, se estimulan por las perturbaciones mecánicas producidas por el latido del corazón del padre o de la madre. Esta estimulación provoca una coordinación entre los parámetros respiratorios, cardiovasculares y térmicos del cuidador y del neonato, siendo este acoplamiento cardio-respiratorio vital para proporcionar un equilibrio óptimo entre el intercambio de gases y las demandas metabólicas ${ }^{37,38}$; esto permite regular parámetros fisiológicos como frecuencia cardiaca, respiratoria, control térmico ${ }^{33,35,39} \mathrm{y}$ crecimiento $^{40}$. Por otra parte, se estimulan los receptores sensoriales de placer que, por la teoría de compuerta, inhiben la conducción de la información de dolor ${ }^{41}$ permitiendo así un control sobre este ${ }^{42-44}$. Asimismo, según la teoría del apego, esta técnica proporciona beneficios a largo plazo ${ }^{45}$ tales como fomentar el vínculo entre padres e hijos, y aliviar el episodio angustioso de haber pasado por un parto prematuro ${ }^{46}$. El papel del personal de salud en este método es guiar la técnica e instruir a la madre sobre la importancia de realizarla con el bebé ${ }^{36,40}$, ya que la continuidad facilita los beneficios anteriormente nombrados ${ }^{37-40,44}$.

Otra práctica que se usa en los neonatos para favorecer un estado de bienestar apoyado en el adecuado desarrollo de la función pulmonar incluye las maniobras de tórax y el empleo de técnicas de digitopresión en los músculos respiratorios, para fomentar el desarrollo de patrones de movimiento torácicos normales $\mathrm{y}$, por lo tanto, alcanzar una respiración eficiente ${ }^{47}$. Las maniobras de tórax tienen 
como objetivos específicos lograr el aclaramiento de la vía aérea, prevenir la retención de esputo, tratar el colapso pulmonar, reducir el riesgo de reintubación, y mejorar la ventilación pulmonar 23,24,48,49. Adicionalmente, la aplicación de las maniobras de tórax favorece la reducción de la atelectasia postextubación cuya principal causa es la hiperoxia producida por la oxigenoterapia, esta se caracteriza por la producción de radicales libres y la subsecuente activación de fagocitos, así como también provoca inflamación y fibrosis, procesos que se relacionan también con riesgo de retinopatía grave del prematuro debido a la susceptibilidad de la retina a los radicales libres ${ }^{50-53}$.

Las maniobras de tórax se pueden clasificar en convencionales y no convencionales. Las maniobras convencionales incluyen posicionamiento, percusión de tórax y vibración realizada en conjunto con la succión para eliminar las secreciones de las vías aéreas centrales ${ }^{23}$. El posicionamiento estabiliza la caja torácica y proporciona la mejor longitud para las fibras musculares del diafragma y mejora su ángulo de contracción. Por lo tanto, se ha asociado con mayores ventajas como aumento en el volumen corriente, mejora en la función del diafragma, reducción en la asincronía toracoabdominal, aumento en la oxigenación y también disminución en la retención del dióxido de carbono ${ }^{54,55}$.

Las técnicas de vibración y percusión producen una oscilación del tórax por compresión o percusión rápida con las manos del fisioterapeuta, encaminadas para mejorar las mediciones respiratorias en cuanto a saturación de oxígeno y frecuencia respiratoria, el tiempo en la oxigenoterapia o la duración de la estancia hospitalaria en los neonatos; esto se logra al favorecer la remoción de secreciones de la vía aérea, así como también ha mostrado un efecto benéfico en cuanto a prevención de atelectasias post extubación ${ }^{16,54,56-59}$. Sin embargo, estas técnicas deben ser aplicadas por un fisioterapeuta con experiencia, monitorizando los signos vitales, así como también se debe tener especial precaución ante posibles efectos adversos dados por una intolerancia a la técnica como taquicardia, taquipnea, o hipoxemia ${ }^{57,58}$.

Por otra parte, se encuentran las maniobras no convencionales, las cuales incluyen técnicas espiratorias forzadas y lentas. Las técnicas espiratorias forzadas consisten en aumentar repentinamente el flujo espiratorio comprimiendo el tórax o el abdomen, y las técnicas de flujo lento consisten en comprimir la caja torácica y la cavidad abdominal de manera gradual y suave desde la fase espiratoria media hasta el final de la espiración ${ }^{16}$. Estas estrategias son útiles para lograr eliminar el exceso de moco y optimizar la ventilación del neonato en $\mathrm{UCl}{ }^{25,56}$. Finalmente, las técnicas tanto de vibración y percusión como de espiración forzada y lenta no se deben aplicar si el neonato presenta inestabilidad hemodinámica, hemorragia intraventricular, y/o fracturas costales 57,58 .

A pesar de la importancia en la UCIN de las estrategias de intervención fisioterapéuticas más usadas, como las técnicas de masaje, estimulación kinestésica, el método madre canguro, la digitopresión y las maniobras de tórax, no hay en la literatura un documento que recopile sus aplicaciones, por lo que el objetivo de este artículo es revisar la información proveniente de la evidencia científica disponible sobre las diferentes estrategias de intervención anteriormente mencionadas, desde un enfoque integral, teniendo en cuenta su aplicación en la unidad de cuidado intensivo neonatal.

\section{Metodología de búsqueda}

La pregunta de investigación abordada fue: ¿Cuál es la información disponible sobre la aplicación y efectos de las modalidades de intervención fisioterapéuticas como masaje, estimulación kinestésica, método madre canguro, digitopresión y maniobras de tórax en los neonatos que se encuentran en la unidad de cuidados intensivos neonatal? El problema de investigación fue tratado aplicando la metodología del sistema PICO (Población, intervención, comparación y "outcome" o resultado). Siguiendo esta estrategia, la población a tratar fueron neonatos que se encuentren en la UCIN; las intervenciones son la aplicación y efectos del masaje, estimulación kinestésica, método madre canguro, maniobras de tórax y digitopresión; la comparación fue con o sin grupo control (placebo); y el resultado esperado fue conocer la aplicación y los efectos de la intervención.

La revisión bibliográfica de artículos fue efectuada entre el 4 de febrero y el 30 de agosto del 2019 en las bases de datos Pubmed y Scopus, realizando 3 grandes búsquedas, una para masaje y estimulación kinestésica, otra para el método madre canguro y una final para maniobras de tórax y digitopresión. Para lo anterior, se utilizaron las palabras claves "Cuidado Intensivo Neonatal", "Masaje", 
"Método madre canguro", "Estimulación Física", "Terapia Respiratoria", "Maniobras de tórax" y "Desarrollo neuromotor". Los criterios de inclusión considerados en general fueron aquellos estudios enfocados en la aplicación y efectos del masaje, la estimulación kinestésica, el método madre canguro, la digitopresión y las maniobras de tórax en los neonatos que se encontraran en la UCIN, adicionalmente se incluyeron artículos que estuvieran completos en las bases de datos, los cuales en su mayoría se encontraron en inglés y algunos en español. Los criterios de exclusión fueron aquellos artículos que se encontraban duplicados, que en su título y/o abstract no nombraran intervenciones en la población neonatal y artículos cuya fecha de publicación fuera inferior al año 2009, este último aspecto se tuvo en cuenta con la finalidad de tener la información más actual y pertinente.

Se escogieron en las bases de datos los artículos con los títulos que más se relacionaran con los términos de búsqueda, posteriormente se seleccionaron los que tuvieran los resúmenes de mayor relación con el tema de esta revisión, y finalmente se eligieron los artículos con base al texto completo. Las 3 búsquedas se consolidaron en una sola búsqueda y se obtuvo el PRISMA global (ver Figura 1).

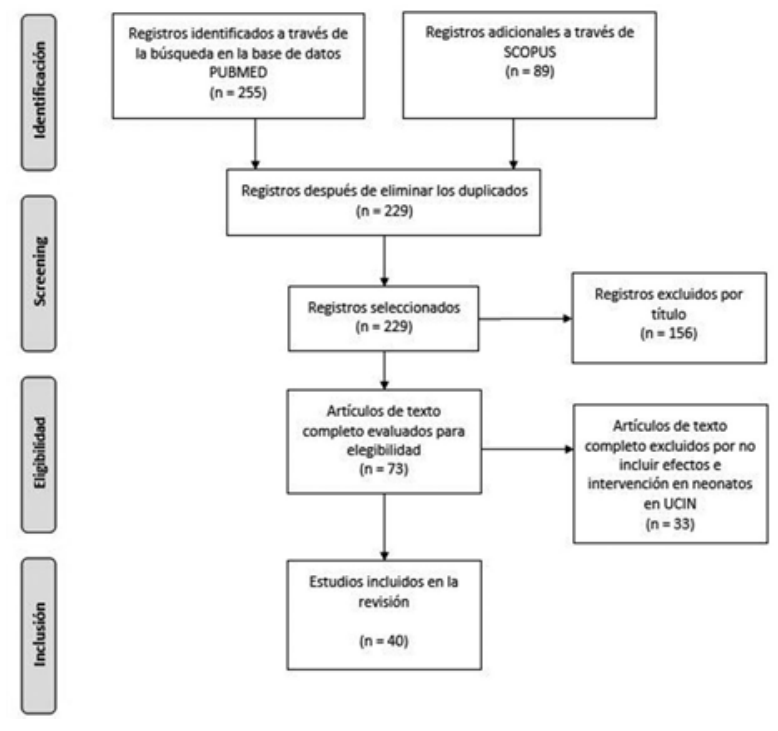

Figura 1. PRISMA Global

Fuente: Modificada por autores

\section{Desarrollo del tema}

A través de la búsqueda, se obtuvieron un total de 344 artículos (ver Figura 1), a los cuales se les aplicaron los criterios de inclusión mencionados, quedando disponibles 73 para revisión metodológica, de los cuales se excluyeron 33 según los criterios, y finalmente se seleccionaron 40 para la elaboración de esta revisión.

\section{Estimulación kinestésica}

Las investigaciones muestran que los programas que incluyen este tipo de intervención benefician a los neonatos con bajo peso al nacer y/o con prematurez, como lo mencionan Oberg et al. ${ }^{29}$ : "a través del movimiento, los bebés aprenden y experimentan sensaciones mediante los cuales se desarrolla la competencia kinestésica. Sobre la base de movimientos espontáneos innatos, el bebé aprende a conocer su propio cuerpo, así como a adquirir conocimiento y comprensión del entorno. Sus cuerpos son tanto expresivos como experimentados al mismo tiempo. Por lo tanto, el desarrollo infantil puede entenderse como resultado de la interacción entre el sistema que consiste en percepción, sensación y movimiento". Involucra el desarrollo motor el cual es un proceso no lineal de retroalimentación basado en la interacción entre diferentes subsistemas en el niño, el entorno y la tarea. Las investigaciones con este tipo de intervención lo que buscan es optimizar el control postural y el movimiento selectivo en los recién nacidos prematuros para reducir el grado de retraso en el desarrollo motor, gracias a la alta plasticidad cerebral del neonato, y desarrollar alta calidad del movimiento y función de los niños prematuros 29 . A su vez, buscan mejorar el aumento de peso, la mineralización ósea, lograr un alta hospitalaria más temprana, obtener respuestas conductuales y motoras más óptimas ${ }^{14}$, reducir el estrés y mejorar los resultados de desarrollo del bebé cuando se aplica de manera adecuada ${ }^{16}$.

Para ello se encontraron varias formas de realizar estimulación kinestésica en esta población. Entre estas está la técnica de facilitación de Kabat del movimiento, en la cual mediante presión/compresión ajustada intermitente sobre grupos musculares y articulaciones relevantes cuando el bebé está en decúbito supino y decúbito prono se ha asociado con mejor desarrollo del control de la cabeza y el tronco en cada posición. El tiempo ideal de duración de esta intervención es hasta diez minutos, dos veces al día, durante un período de tres semanas; sin embargo, esta duración se ajusta según la respuesta y el estado del bebé, ya que si este muestra algún signo de incomodidad o estrés como cambios en el color 
de la piel, respiración irregular, cambios no deseados en el tono muscular o movimientos incontrolados, se suspende la sesión. Finalmente se recomienda realizarla media hora a dos horas antes o después de la comida, para evitar reflejos vaso vágales ${ }^{29}$.

El otro tipo de estimulación kinestésica fue desarrollada con movimientos de cada extremidad, teniendo como puntos de contacto con el bebé en miembros superiores brazo y codo, y en miembros inferiores pierna y rodilla. Una vez esto, se procede a realizar ejercicios de flexo-extensión en las articulaciones de muñeca, codo, hombro, tobillo, rodilla y cadera ${ }^{11,14,16}$. Este tipo de intervención logró una mejora significativa del peso en los grupos que fueron sometidos a la terapia y permitió reducir el tiempo de estancia hospitalaria"11, así como mejorar el puntaje del "Test of Infant Motor Performance" en el estudio en que fue evaluado; de igual manera, se correlacionó con una disminución en el tiempo con nutrición parenteral total cuando se combinaba con masaje $^{16}$.

\section{Masaje}

Los estudios encontrados en esta revisión de la literatura usan intervenciones indicadas para estimular a los neonatos a término, pretérmino y/o con bajo peso al nacer que se encontraran en la UCIN o en hospitalización, y que tuvieran diferentes aceites de masaje, tales como girasol, triglicéridos, oliva y mineral ${ }^{11,15,17,20}$, así como que también tuvieran diferentes técnicas y objetivos de aplicación. Una de las técnicas originales publicadas por Field et al fue posteriormente modificada por 2 fisioterapeutas, dando como resultado una terapia consistente en 2 repeticiones de una serie de trazos definidos a 5 zonas del cuerpo. El masaje se realizó en intervalos de 1 minuto, con la aplicación de 12 golpeteos que duran aproximadamente 5 segundos cada uno para cada una de las áreas del cuerpo que reciben masaje: en prono en cabeza y cuello, cuello y hombros, dorso superior a inferior, muslo a pie y hombro a manos, en ese orden secuencial ${ }^{17}$.Esta terapia no evidenció diferencias significativas en la calidad del sueño entre el grupo intervención y el control, sin embargo permitió mantener estabilidad hemodinámica ${ }^{17}$. En otros estudios que siguieron la técnica de Field et al. no se produjeron reacciones adversas fisiológicas ${ }^{16}$, sino que se obtuvo una ganancia significativa de peso ${ }^{11}$, un periodo significativamente menor de estancia en unidad de cuidado intensivo neonatal ${ }^{14}$, puntuaciones del "Premature Infant Pain Profile" significativamente más bajas después de la terapia y del alta en comparación con el grupo control'5, y puntaje del "Test of Infant Motor Performance" de salida con una mejoría significativa respecto al grupo control en el estudio en que fue evaluado ${ }^{16}$.

El masaje combinado con estimulación kinestésica ( 5 minutos de masaje - 5 minutos de estimulación kinestésica - retorno al masaje 5 minutos, 5:5:5) con patrón cefalocaudal y leve presión en las articulaciones, evidenció aumentar la citotoxicidad de las células $\mathrm{NK}^{18}$, esta función es la más conocida de éstas células y la ejercen sobre diferentes tipos celulares: células tumorales, transformadas por virus, infectadas con bacterias y otros patógenos, lo que les confiere un amplio papel defensivo frente a enfermedades neoplásicas e infecciosas ${ }^{60}$. Este tipo de masaje también evidenció un aumento significativo del peso respecto a terapia de solo masaje ${ }^{20}$. Por otra parte, el masaje realizado con patrón cefalocaudal, con golpeteos y con aceite de girasol durante 10 minutos, evidenció aumentar de forma significativa el peso a los 28 días en el grupo de masaje con aceite respecto al control ${ }^{12}$.

\section{Método Madre Canguro}

En esta categoría de intervención se revisa la importancia del rol del fisioterapeuta en la educación a padres y familiares sobre la implementación de esta técnica en los neonatos prematuros, en otras palabras, aquellos que nacen antes de las 37 semanas de gestación o que presenten bajo peso al nacer con un peso inferior a 2500 gramos independiente de la edad gestacional ${ }^{32}$. En la educación a los padres y familiares se debe brindar la información sobre los beneficios respecto a la regulación de parámetros vitales como la frecuencia respiratoria, frecuencia cardiaca, temperatura y el crecimiento gracias al acople cardio-respiratorio ${ }^{38}$, así como tener en cuenta los beneficios en cuanto al apego y vínculo emocional con el cuidador que trae la aplicación de este método para el bebé y su familia ${ }^{37,61}$. En el estudio de Rangey et al., se comparó el masaje con la técnica 5:5:5 versus la implementación de la posición canguro sobre las variables fisiológicas, y se descubrió que las dos son igual de efectivas para mejorar peso y mantener estabilidad fisiológica y hemodinámica ${ }^{61}$.

En el estudio de Bera et al., se brindó educación y orientación a madres residentes en la India sobre la lactancia materna exclusiva y los beneficios del método madre canguro en neonatos que se 
encontraban en la UCIN, los bebés eran vestidos solo con una gorra, calcetines y pañal. Se colocó al bebé en posición canguro, se alentó a la madre a mantener contacto visual con el bebé, y se le permitió succionar el seno con la frecuencia que quisiera, pero al menos cada 2 horas, vigilando continuamente que los parámetros fisiológicos vitales como la frecuencia cardiaca, frecuencia respiratoria, saturación y temperatura se mantuvieran estables durante la lactancia, además de realizar un adecuado seguimiento al peso corporal del neonato para prevenir efectos adversos debido al esfuerzo físico que podía demandar el succionar. De igual manera, se alentó a ir aumentando el tiempo de contacto piel con piel hasta que la madre pudiera proporcionar 1216 horas al día esta posición, esto siempre y cuando hubiera estabilidad en cuanto a los parámetros fisiológicos y en cuanto al peso corporal del neonato con el objetivo de que continuarán esta intervención hasta las 40 semanas de edad corregida o $2500 \mathrm{~g}$ de peso corporal. Esta educación y seguimiento de instrucciones por parte de las madres permitió lograr un desarrollo tanto mental como motor significativamente mejor en los lactantes de posición canguro que en los controles, y mejor desarrollo mental y motor a los 12 meses de seguimiento ${ }^{34}$.

En otro estudio se realizó una división de la población, por una parte, los bebés más prematuros con educación en el programa madre canguro y por otro lado los bebés prematuros tardíos (mayor de 34 semanas de gestación) o a término sin intervención. Después de lograr las 40 semanas en los bebés pretérmino de tratamiento, y tras el seguimiento en casa de estos a los 3, 6, 9 y 12 meses, se evaluó la escala del comportamiento del infante pretérmino y se obtuvo que los neonatos prematuros se compararon con los a término en las siguientes variables: organización fisiológica, organización motora, y organización de atención ${ }^{62}$.

En el estudio de Azevedo y colaboradores se evidenció en prematuros intubados con peso menor a $1500 \mathrm{~g}$ que se encontraban en la UCIN de estabilidad fisiológica y hemodinámica, una significativa reducción de la $\mathrm{FIO}_{2}$ en la posición canguro y una significativa reducción del dolor según la escala "Premature Infant Pain Profile" et al, no fue significativamente diferente la atención con el programa madre canguro que la atención convencional (alzado con cobija), sin embargo, las dos demostraron aumentar de peso y aumentar la tasa de supervivencia del neonato pretérmino ${ }^{40}$.

\section{Maniobras de tórax convencionales}

Esta intervención fue aplicada por el estudio de Mehta et al. a neonatos que se encontraban en la UCIN que presentaban estertores, roncus 0 disminución de los sonidos respiratorios en la auscultación y que tuvieran Ventilación Mandatoria Intermitente Sincronizada, Presión Positiva Continua en las vías Respiratorias o que tuvieran medidas de oxigenoterapia de alto flujo, como cánula nasal de alto flujo, o de medidas de bajo flujo, como cánula nasal convencional. El drenaje postural se aplicó durante 10 minutos a todos los bebés según las zonas de drenaje, dadas por el origen de la alteración, y en esta posición se realizó percusión y vibración de forma intermitente para movilizar secreciones a vías aéreas centrales. La saturación de oxígeno aumentó significativamente después de estimular la vía aérea y succionar, lo que se relacionó con una ausencia significativa en la crepitación a la auscultación. Por otro lado, hubo una mejora significativa tanto en los resultados radiográficos y en el gradiente de $\mathrm{PaO}_{2}$ / FiO2 y A-aDO2 como en la mejora post terapia de tórax en el grupo de la ventilación mecánica ${ }^{64}$. Asimismo, es importante tener en cuenta que el drenaje postural consiste en colocar al neonato en una posición que emplee la gravedad para mover el moco desde la unidad pulmonar que se encuentre con secreciones a las zonas centrales de la vía aérea donde se expectoran más fácilmente; se encuentran 12 diferentes posiciones para realizarlo, 1 por cada segmento pulmonar, su aplicación y la zona a drenar depende de la auscultación y los hallazgos radiográficos. Adicionalmente de la presencia de estabilidad hemodinámica, el neonato a su vez no debe cursar con hemorragia intraventricular de grado tres y superior ${ }^{54,58,59,65}$, alteraciones de la presión venosa central, no debe tener fracturas costales 0 vertebrales, ni cirugías recientes ${ }^{66}$. Finalmente, se debe realizar un monitoreo continuo de los signos vitales si el neonato cumple con los criterios para realizar el drenaje, y asimismo, se debe tener especial precaución ante posibles efectos adversos dados por una intolerancia a la técnica como taquicardia, taquipnea, o hipoxemia ${ }^{57,58}$

En el estudio de Shannon et al. se indagó la efectividad de las técnicas convencionales de tórax en neonatos e infantes con ventilación mecánica en UCIN, pero estables hemodinámicamente, dependiendo de si la técnica era realizada por un fisioterapeuta de guardia o uno especializado, y se encontró una caída significativa de la resistencia respiratoria en 
ambos grupos de tratamiento de fisioterapia, que se mantuvo significativa 30 minutos después. Hubo mejoras clínicamente importantes en los resultados respiratorios después de 27 (43\%) tratamientos realizados por fisioterapeutas de guardia en comparación con 40 (63\%) tratamientos realizados por terapeutas especializados, la diferencia fue estadísticamente significativa ${ }^{67}$.

Finalmente, la revisión sistemática de Chaves et al. comparó la fisioterapia torácica convencional con el posicionamiento lateral para el mejoramiento del intercambio gaseoso durante cinco minutos, una vez al día, durante toda la estancia hospitalaria en neonatos en el límite del primer mes de vida y en infantes con neumonía. Se encontró que no hubo diferencias significativas en los estudios de Lukrafka et al. y Paludo et al. en la duración de la hospitalización entre los grupos control e intervención, sin embargo, en ambos estudios hubo diferencias en la frecuencia de respiración a favor del grupo de intervención. El estudio de Zhao consideró solo los niveles de saturación de oxígeno periférico e informó que el grupo de intervención había mejorado estos niveles después de la aplicación de una presión positiva continua en la vía aérea en comparación con el grupo de control ${ }^{68}$.

\section{Maniobras de tórax no convencionales}

En la revisión sistemática de Roque et al. se exploró la eficacia de las técnicas espiratorias pasivas lentas en comparación con ninguna intervención en infantes hospitalizados con bronquiolitis aguda ${ }^{69}$. En neonatos sin asistencia respiratoria dada por ventilación mecánica, los ensayos incluidos mostraron un beneficio significativo de las técnicas de fisioterapia en el cambio de la gravedad de la enfermedad, los parámetros de respuesta, la duración de la estancia hospitalaria o los requisitos de oxígeno en esta población ${ }^{69}$. Un ensayo encontró mejoras transitorias inmediatas en la puntuación respiratoria en pacientes con bronquiolitis moderada que recibieron técnicas espiratorias lentas ${ }^{70}$. Los ensayos incluidos no informaron eventos adversos graves, sin embargo el estudio de Gajdos et al. informó un significativo número de vómitos en el procedimiento $^{71}$.

Finalmente, el estudio de Postiaux et al. comparó la efectividad entre las técnicas convencionales de tórax con las no convencionales en neonatos y lactantes con bronquiolitis aguda para la mejora de los parámetros respiratorios y encontró que los días de hospitalización fueron similares entre los grupos. La puntuación clínica de Wang para clasificar la bronquiolitis se redujo significativamente en los dos grupos de intervención, la dificultad respiratoria durante este período disminuyó y después de 72 horas se observó significativa reducción en la puntuación clínica de Wang sólo para los pacientes tratados con maniobras no convencionales ${ }^{72}$.

\section{Conclusiones}

De acuerdo con esta revisión, se puede concluir que hay una amplia variedad de prácticas realizadas por el fisioterapeuta y otros profesionales en salud, tales como masaje, estimulación kinestésica, educación en posición canguro, digitopresión y maniobras de tórax, cuyo fin último es lograr el adecuado desarrollo fisiológico y neuromotor del neonato a lo largo de su vida, siendo el masaje la intervención más practicada debido a sus potenciales efectos sobre las variables fisiológicas del neonato. En cuanto a la estimulación kinestésica se encuentra una gran efectividad respecto al logro de un adecuado desarrollo neuromotor, mineralización ósea, y disminución del estrés, evidenciando aún mayores efectos si es combinado con masaje en modo 5:5:5. Con respecto a la educación en el método madre canguro se observa que es una estrategia fundamental, ya que permite el acople cardio-respiratorio entre madre e hijo, además de favorecer el apego y el vínculo de los cuidadores con el neonato. Finalmente, en cuanto a la efectividad de las técnicas de tórax, la evidencia actual sustenta su uso en casos de ocupación bronquial o alveolar, y para disminuir el riesgo de atelectasias post extubación, sin embargo, en otras condiciones la evidencia es insuficiente para extrapolar a la práctica clínica.

\section{Referencias bibliográficas}

1. Patra Barbosa A, Johnston C, Brunow de Carvalho W. Fisioterapia pediátrica y neonatal. Distribuna; 2012.

2. Ministerio de Salud y Protección Social: Dirección de Epidemiología y Demografía. Análisis de Situación de Salud (ASIS) Colombia, 2018. [internet] Bogotá D.C., Colombia; 2019. Disponible en: https:/www.minsalud.gov.co/sites/rid/Lists/ BibliotecaDigital/RIDE/VS/ED/PSP/asis-colombia-2018.pdf

3. Marrugo-Arnedo CA, Arrieta-Arrieta A, Herrera-Malambo D, DíazVargas LC, Pérez-Yepes C, Dueñas-Castell C, et al. Determinantes de estancia prolongada de neonatos en una unidad de cuidados intensivos. Rev. cienc. salud (Bogotá). 2019;17(2):259-75.

4. Peña CB, Pinzón YA, Forero YJ, Pantoja JA, Giraldo LF, Bastidas $\mathrm{AR}$, et al. Características de pacientes ingresados a la unidad de cuidado intensivo neonatal en la Clínica Universidad de La Sabana. Rev. Univ. Ind. Santander, Salud. 2016;48(4):480-5.

5. Chokshi T, Alaparthi GK, Krishnan S, Vaishali K, Zulfeequer CP. Practice patterns of physiotherapists in neonatal intensive care 
units: A national survey. Indian J Crit Care Med. 2013;17(6):359-66.

6. Byrne E, Garber J. Physical therapy intervention in the neonatal intensive care unit. Phys Occup Ther Pediatr. 2013;33(1):75-110.

7. Sweeney JK, Heriza CB, Blanchard Y. Neonatal physical therapy. Part I: Clinical competencies and neonatal intensive care unit clinical training models. Pediatr Phys Ther. 2009;21(4):296-307.

8. Sweeney JK, Heriza CB, Blanchard Y, Dusing SC. Neonatal physical therapy. Part II: Practice frameworks and evidencebased practice guidelines. Pediatr Phys Ther. 2010;22(1):2-16.

9. Byrne E, Campbell SK. Physical therapy observation and assessment in the neonatal intensive care unit. Phys Occup Ther Pediatr. 2013;33(1):39-74

10. Álvarez MJ, Fernández D, Gómez-Salgado J, Rodríguez-González D, Rosón M, Lapeña S. The effects of massage therapy in hospitalized preterm neonates: A systematic review. Int J Nurs Stud. 2017;69:119-36

11. Taheri PA, Goudarzi Z, Shariat M, Nariman S, Matin EN. The effect of a short course of moderate pressure sunflower oil massage on the weight gain velocity and length of NICU stay in preterm infants. Infant Behav Dev. 2018;50:22-7.

12. Kumar J, Upadhyay A, Dwivedi AK, Gothwal S, Jaiswal V, Aggarwal S. Effect of oil massage on growth in preterm neonates less than $1800 \mathrm{~g}$ : a randomized control trial. Indian J Pediatr. 2013;80(6):465-9.

13. World Health Organization: WHO. Nacimientos prematuros [Internet]. Who.int. World Health Organization: WHO; 2018. Disponible en: https://www.who.int/es/news-room/fact-sheets/ detail/preterm-birth\#: : :text $=$ Los\%20ni\%C3\%B1os\%20 prematuros\%20se\%20dividen,tard\%C3\%ADos\%20(32\%20a\%20 $37 \% 20$ semanas

14. Massaro AN, Hammad TA, Jazzo B, Aly H. Massage with kinesthetic stimulation improves weight gain in preterm infants. J Perinatol. 2009;29(5):352-7.

15. Abdallah B, Badr LK, Hawwari M. The efficacy of massage on short and long term outcomes in preterm infants. Infant Behav Dev. 2013;36(4):662-9.

16. Ho YB, Lee RSY, Chow CB, Pang MYC. Impact of massage therapy on motor outcomes in very low-birthweight infants: randomized controlled pilot study. Pediatr Int. 2010;52(3):378-85.

17. Yates CC, Mitchell AJ, Booth MY, Williams DK, Lowe LM, Whit Hall R. The effects of massage therapy to induce sleep in infants born preterm. Pediatr Phys Ther. 2014;26(4):405-10.

18. Ang JY, Lua JL, Mathur A, Thomas R, Asmar BI, Savasan $\mathrm{S}$, et al. A randomized placebo-controlled trial of massage therapy on the immune system of preterm infants. Pediatrics. 2012;130(6):e1549-58.

19. Choi HJ, Kim SJ, Oh J, Lee MN, Kim SH, Kang KA. The effects of massage therapy on physical growth and gastrointestinal function in premature infants: A pilot study. J Child Health Care. 2016;20(3):394-404.

20. Saeadi R, Ghorbani Z, Shapouri Moghaddam A. The effect of massage with medium-chain triglyceride oil on weight gain in premature neonates. Acta Med Iran. 2015;53(2):134-8.

21. Field $T$, Diego $M$, Hernandez-Reif $M$. Potential underlying mechanisms for greater weight gain in massaged preterm infants. Infant Behav Dev. 2011;34(3):383-9.

22. Allen KA. Promoting and protecting infant sleep. Adv Neonatal Care. 2012;12(5):288-91.

23. Smith SL, Lux R, Haley S, Slater H, Beachy J, Beechy J, et al. The effect of massage on heart rate variability in preterm infants. J Perinatol. 2013;33(1):59-64.

24. Vignochi CM, Teixeira PP, Nader SS. Effect of aquatic physical therapy on pain and state of sleep and wakefulness among stable preterm newborns in neonatal intensive care units. Rev Bras Fisioter. 2010 May-Jun;14(3):214-20.

25. McGrath JM. Touch and massage in the newborn period: effects on biomarkers and brain development. J Perinat Neonatal Nurs. 2009;23(4):304-6

26. Lobo MA, Harbourne RT, Dusing SC, McCoy SW. Grounding early intervention: physical therapy cannot just be about motor skills anymore. Phys Ther. 2013;93(1):94-103.

27. Tekgündüz KŞ, Gürol A, Apay SE, Caner I. Effect of abdomen massage for prevention of feeding intolerance in preterm infants. Ital J Pediatr. 2014;40:89

28. Pepino VC, Mezzacappa MA. Application of tactile/kinesthetic stimulation in preterm infants: a systematic review. J Pediatr (Rio
J). 2015;91(3):213-33.

29. Oberg GK, Campbell SK, Girolami GL, Ustad T, Jørgensen L, Kaaresen PI. Study protocol: an early intervention program to improve motor outcome in preterm infants: a randomized controlled trial and a qualitative study of physiotherapy performance and parental experiences. BMC Pediatr. 2012;12:15.

30. Puccini FRS, Berretin-Felix G. Gastroesophageal reflux and swallowing in newborns and infants: Integrative review of literature. Rev CEFAC. 2015;17(5):1664-73.

31. Rameshkumar R, Bansal A. Approach and Management of Children with Raised Intracranial Pressure. J Pediatr Crit Care. 2015;2:13.

32. Charpak N, Ruiz JG, Angel MI, Duque JS, Garcia C. Lineamientos tecnicos para la implementacion de Programas Madre Canguro en Colombia. Ministerio de Proteccion Social Republica de Colombia; 2009.

33. Zhang SH, Yip WK, Lim PF, Goh MZ. Evidence utilization project: implementation of kangaroo care at neonatal ICU. Int J Evid Based Healthc. 2014;12(2):142-50.

34. Bera A, Ghosh J, Singh AK, Hazra A, Mukherjee S, Mukherjee R. Effect of kangaroo mother care on growth and development of low birthweight babies up to 12 months of age: a controlled clinical trial. Acta Paediatr. 2014;103(6):643-50.

35. Davanzo R, Brovedani P, Travan L, Kennedy J, Crocetta A, Sanesi C, Strajn T, De Cunto A. Intermittent kangaroo mother care: a NICU protocol. J Hum Lact. 2013;29(3):332-8.

36. Callister LC. Kangaroo mother care for preterm infants globally. MCN Am J Matern Child Nurs. 2015;40(3):198.

37. Head LM. The effect of kangaroo care on neurodevelopmental outcomes in preterm infants. J Perinat Neonatal Nurs. 2014;28(4):290-9; quiz E3-4.

38. Bloch-Salisbury E, Zuzarte I, Indic P, Bednarek F, Paydarfar D. Kangaroo care: cardio-respiratory relationships between the infant and caregiver. Early Hum Dev. 2014;90(12):843-50.

39. Baley J; COMMITTEE ON FETUS AND NEWBORN. Skin-toSkin Care for Term and Preterm Infants in the Neonatal ICU. Pediatrics. 2015;136(3):596-9.

40. Ghavane S, Murki S, Subramanian S, Gaddam P, Kandraju H, Thumalla S. Kangaroo Mother Care in Kangaroo ward for improving the growth and breastfeeding outcomes when reaching term gestational age in very low birth weight infants. Acta Paediatr. 2012;101(12):e545-9.

41. Hatfield LA. Neonatal pain: What's age got to do with it? Surg Neurol Int. 2014;5(Suppl 13):S479-89.

42. Cong X, Cusson RM, Hussain N, Zhang D, Kelly SP. Kangaroo care and behavioral and physiologic pain responses in very-low-birthweight twins: a case study. Pain Manag Nurs. 2012;13(3):127-38.

43. Zhang F, Liu S. Kangaroo mother care may help oral growth and development in premature infants. Fetal Pediatr Pathol. 2012;31(4):191-4.

44. Baird D, Bybel M. Effectiveness of Skin-to-Skin Care for Procedure-Related Pain in Newborns. Am Fam Physician. 2018;97(3):170-1.

45. Carducci B, Bhutta ZA. Care of the growth-restricted newborn. Best Pract Res Clin Obstet Gynaecol. 2018;49:103-16.

46. Samra HA, Dutcher J, McGrath JM, Foster M, Klein L, Dira G, et al. Effect of Skin-to-Skin Holding on Stress in Mothers of LatePreterm Infants: A Randomized Controlled Trial. Adv Neonatal Care. Adv Neonatal Care. 2015;15(5):354-64.

47. Giannantonio C, Papacci P, Ciarniello R, Tesfagabir MG, Purcaro V, Cota F, et al. Chest physiotherapy in preterm infants with lung diseases. Ital J Pediatr. 2010;36:65.

48. Sant'Anna GM, Keszler M. Weaning infants from mechanical ventilation. Clin Perinatol. 2012;39(3):543-62.

49. de Abreu LC, Valenti VE, de Moura Filho OF, Vanderlei LCM, de Carvalho TD, Vertamatti MAF, et al. Chest associated to motor physiotherapy acutely improves oxygen saturation, heart rate and respiratory rate in premature newborns with periventricularintraventricular hemorrhage. HealthMED. 2011;5(6):1381-8.

50. Perrone S, Bracciali C, Di Virgilio N, Buonocore G. Oxygen use in neonatal care: a two-edged sword. Front Pediatr. 2016;4:143.

51. Santos AK, Silveira J, Neves VC, Zotz TGG, Motter AA, Andreazza MG. Atelectasis and lung changes in preterm neonates in the neonatal period: a blind radiological report and clinical findings. Rev Bras Ter Intensiva. 2019;31(3):347-53.

52. de Paula LCS, Siqueira FC, Juliani RCTP, de Carvalho WB, Ceccon 
MEJR, Tannuri U. Post-extubation atelectasis in newborns with surgical diseases: a report of two cases involving the use of a highflow nasal cannula. Rev Bras Ter Intensiva. 2014;26(3):317-20.

53. Dominguez MC, Alvares BR. Pulmonary atelectasis in newborns with clinically treatable diseases who are on mechanical ventilation: clinical and radiological aspects. Radiol bras. 2018;51(1):20-5

54. Walsh BK, Hood K, Merritt G. Pediatric airway maintenance and clearance in the acute care setting: how to stay out of trouble. Respir Care. 2011; 56(9):1424-40;discussion 1440-4.

55. Brunherotti MAA, Martinez FE. Response of oxygen saturation in preterm infants receiving rib cage stabilization with an elastic band in two body positions: a randomized clinical trial. Braz J Phys Ther. 2013;17(2):105-11.

56. Pandya YS, Shetye J, Nanavati R, Mehta A. Resolution of lung collapse in a preterm neonate following chest physiotherapy. Indian J Pediatr. 2011;78(9):1148-50.

57. Goldsmith JP, Karotkin E, Suresh G, Keszler M. Assisted ventilation of the neonate E-book. 6th ed. Elsevier Health Sciences; 2016.

58. Christian PS. Chest physiotherapy for infants. Int J Physiother Res. 2014;2(5):699-705.

59. Rocha G, Soares P, Gonçalves A, Silva AI, Almeida D, Figueiredo S, et al. Respiratory Care for the Ventilated Neonate. Can Respir J. 2018;2018:7472964

60. Sánchez IP, Leal-Esteban LC, Orrego-Arango JC, Garcés-Samudio CG, Gómez-Arias RD, Franco JL, et al. Variaciones en el número y función de los linfocitos asesinos naturales durante infecciones recurrentes o graves. Biomédica. 2014;34(1):118-31.

61. Rangey PS, Sheth M. Comparative Effect of Massage Therapy versus Kangaroo Mother Care on Body Weight and Length of Hospital Stay in Low Birth Weight Preterm Infants. Int J Pediatr. 2014;2014:434060.

62. Neu M, Robinson J, Schmiege SJ. Influence of holding practice on preterm infant development. MCN Am J Matern Child Nurs. 2013;38(3):136-43.

63. Azevedo VMG de O, Xavier CC, Gontijo F de O. Safety of
Kangaroo Mother Care in intubated neonates under 1500 g. J Trop Pediatr. 2012;58(1):38-42.

64. Mehta Y, Shetye J, Nanavati R, Mehta A. Physiological effects of a single chest physiotherapy session in mechanically ventilated and extubated preterm neonates. J Neonatal Perinatal Med. 2016;9(4):371-6.

65. DiBlasi R, Gallagher JT. 27 - Respiratory Care of the Newborn. In: Goldsmith JP, Karotkin EH, Keszler M, Suresh GK. Assisted Ventilation of the Neonate. 6th ed. Elsevier Health Sciences; 2016. p. 291-309.e4.

66. West MP. Chapter 22 - Postural Drainage. En: C. Paz, JC. \& West, MP. (Eds). Acute Care Handbook for Physical Therapists. $4^{\circ}$ ed. St. Louis: W.B. Saunders; 2014. 467-70.

67. Shannon H, Stocks J, Gregson RK, Dunne C, Peters MJ, Main E. Clinical effects of specialist and on-call respiratory physiotherapy treatments in mechanically ventilated children: A randomised crossover trial. Physiotherapy. 2015;101(4):349-56.

68. Chaves GS, Fregonezi GAF, Dias FA, Ribeiro CT, Guerra RO, Freitas DA, et al. Chest physiotherapy for pneumonia in children. Cochrane Database Syst Rev. 2013;(9):CD010277.

69. Roqué i Figuls M, Giné-Garriga M, Granados Rugeles C, Perrotta C, Vilaró J. Chest physiotherapy for acute bronchiolitis in paediatric patients between 0 and 24 months old. Cochrane Database Syst Rev. 2016;2(2):CD004873.

70. Postiaux G, Maffei P, Villiot-Danger JC, Dubus JC. La kinésithérapie respiratoire dans la bronchiolite virale aiguë du nourrisson. Arguments pour/contre [Respiratory physiotherapy in acute viral bronchiolitis in the newborn. Pro/con arguments] Rev Mal Respir. 2018;35(4):403-15.

71. Gajdos V, Katsahian S, Beydon N, Abadie V, De Pontual L, Larrar S, et al. Effectiveness of chest physiotherapy in infants hospitalized with acute bronchiolitis: a multicenter, randomized, controlled trial. PLoS Med. 2010;7(9):e1000345.

72. Gomes EL, Postiaux G, Medeiros DR, Monteiro KK, Sampaio LM, Costa D. Chest physical therapy is effective in reducing the clinical score in bronchiolitis: randomized controlled trial. Rev Bras Fisioter. 2012;16(3):241-7. 